\section{ZUBIRI Y PRIGOGINE: CONSIDERACIONES EN TORNO A LA VIDA, EL DINAMISMO Y LA
AUTOORGANIZACIÓN}

\author{
Ronald Durán Allimant \\ Pontificia Universidad Católica de Valparaíso, Chile \\ Universidad de Playa Ancha, Chile \\ ronald.duran@upla.cl \\ Ricardo Espinoza Lolas \\ Pontificia Universidad Católica de Valparaíso, Chile \\ respinoz@ucv.cl
}

Cómo citar este artículo/Citation: Durán Allimant, R. y Espinoza Lolas, R. (2016). Zubiri y Prigogine: consideraciones en torno a la vida, el dinamismo y la autoorganización. Arbor, 192 (780): a328. doi: http://dx.doi.org/10.3989/arbor.2016.780n4004

Recibido: 25 julio 2014. Aceptado: 01 septiembre 2015.

RESUMEN: Zubiri concibe el ser vivo como estructura dinámica (1968). Prigogine por su parte lo concibe desde la perspectiva de las estructuras disipativas (1967), que aparecen en procesos de autoorganización en sistemas abiertos inestables. Ambas concepciones son prácticamente contemporáneas, y ambas conjugan dos aspectos de lo vivo en cierta medida contradictorios: lo dinámico y lo estructural. Teniendo como base lo dicho por Zubiri respecto al dinamismo de la mismidad en Estructura dinámica de la realidad, y la teoría de estructuras disipativas y procesos de autoorganización de Prigogine, analizaremos algunos puntos centrales de ambas concepciones de lo vivo con el objetivo de comprender sus elementos comunes y sus diferencias principales.

PALABRAS CLAVE: Zubiri; Prigogine; vida; dinamismo; estructura; autoorganización.

\section{ZUBIRI AND PRIGOGINE: CONSIDERATIONS ON LIFE, DYNAMISM AND SELF- ORGANIZATION}

Copyright: (C) 2016 CSIC. Este es un artículo de acceso abierto distribuido bajo los términos de la licencia Creative Commons Attribution (CC BY) España 3.0.

ABSTRACT: Zubiri conceives the living being as a dynamic structure (1968). Prigogine, for his part, conceives it from the perspective of dissipative structures that appear in self-organizing processes in unstable open systems. Both conceptions are practically contemporary, and both unite two aspects of living that are somewhat contradictory: the dynamical and the structural. On the basis of Zubiri's considerations about the dynamism of selfhood in the Dynamic Structure of Reality, and Prigogine's theory of dissipative structures and selforganization processes, we analyze some central points of both concepts of living to understand their common elements and key differences.

KEYWORDS: Zubiri; Prigogine; life; dynamism; structure; selforganization. 


\section{INTRODUCCIÓN}

Casi de manera contemporánea, Zubiri y Prigogine plantean dos concepciones de lo vivo que intentan conjugar en su unidad las dimensiones, en cierta medida opuestas, de lo vivo: lo estructural y lo dinámico. Zubiri en sus lecciones de 1968, denominadas «Estructura dinámica de la realidad», publicadas posteriormente como libro (Zubiri, 2006), se refiere al ser vivo como estructura dinámica. Prigogine, por su parte, a partir de 1967 concibe lo vivo desde la perspectiva de lo que denomina estructuras disipativas en procesos de auto-organización. Sin existir influencia mutua, ambos recogen conceptos y teorías científicas propias de su época. En el presente artículo analizaremos de manera comparada estas propuestas, poniendo de relieve sus aspectos comunes y sus diferencias principales, y el contexto común en el que se elaboran. Para cumplir nuestro objetivo, consideraremos como base lo dicho por Zubiri en Estructura dinámica de la realidad, y en textos de la misma época, sin realizar la "actualización» desde Inteligencia sentiente, tomando la exposición de Estructura dinámica de la realidad como una teoría metafísica, es decir como perteneciente al ámbito de la razón (Zubiri, 1980/2001, p. 39$)^{2}$. Respecto a Prigogine, estudiaremos su teoría principalmente desde la perspectiva de finales de los años 60 y principios de los 70, enfocando su trabajo científico desde una perspectiva filosófica.

\section{LOS SERES VIVOS Y SU ESTABILIDAD DINÁMICA O DINAMISMO DE ESTABILIDAD}

En Estructura dinámica de la realidad, Zubiri identifica tres tipos de dinamismo: a) dinamismo de la variación, b) dinamismo de la alteración, y c) dinamismo de la mismidad. Cada uno está sostenido por el otro, siendo el más básico el de la variación (Zubiri, 2006, p. 158). Éste afecta puramente a las notas adherentes, por ejemplo el lugar que ocupa una cosa en el espacio (Zubiri, 2006, p. 105). El de la alteración, por su parte, implica la producción de una sustantiva otra, de un alter. Aquí encontramos la génesis o evolución (Zubiri, 2006, p. 129). En el último, el dinamismo de la mismidad, Zubiri examina el ser vivo como estabilidad dinámica (Zubiri, 2006, p. 158) ${ }^{3}$. En éste dinamismo nos centraremos comparándolo con las ideas de estructuras disipativas y autoorganización en Prigogine.

Zubiri se refiere al "dinamismo de la mismidad» en estos términos: «Se trata de un dinamismo que aparece en cierto tipo de estructuras sustantivas; aquellas que son estructuralmente estables [...] se trata de una estabilidad propiamente hablando dinámica. Es decir, de la estabilidad de unas sustantividades que, efectivamente, por su dinamismo continúan siendo las mismas en una forma o en otra» (Zubiri, 2006, p. 161). Las sustantividades dinámicamente estables pueden ser de dos tipos: la sustantividad molecular (Zubiri, 2006, p. 162), y la sustantividad transmolecular (Zubiri, 2006, p. 164). La primera se refiere a la estabilidad de las moléculas de diverso tipo y tamaño, que implican una persistencia estructural (Zubiri, 2006, p. 163). El dinamismo trans-molecular, "donde "trans" significa "sistema" de moléculas» (Zubiri, 2006, p. 164), implica la realización de actividades que involucran un sistema de moléculas para mantener la propia estructura o identidad estructural. Este es el dinamismo propio de lo vivo: "No hay ningún ser vivo que sea una sola molécula. $Y$ en este sentido es transmolecular» ( $\mathrm{Zu}$ biri, 2006, p. 165). Los seres vivos no son conjunción, adición o mero acoplamiento de moléculas, sino «sistemas de moléculas» (Zubiri, 2006, p. 165). De esta manera, la estabilidad de un ser vivo no es mera persistencia (como la de un electrón, por ejemplo), sino que «ejerce una enorme actividad precisamente para persistir en su propia sustantividad, en su propia identidad de sustantividad» (Zubiri, 2006, p. 165). Zubiri identifica de la siguiente manera los caracteres del ser vivo como estabilidad o equilibrio dinámico: «No solamente existe algo estable sino que hay una estabilidad para la estabilidad misma. Lo cual significa, primero: que la estructura viva adquiere, en cierto modo, el carácter de una distancia activa, que cuanto más viva sea más se separa del resto del Universo [...] Y en ese mantener su propia estructura sustantiva quiere el ser viviente, en una o en otra forma, independizarse muy relativamente [...] de las vicisitudes que acontecen en torno a él. Segundo: esta estructura es respectiva. $Y$ aquí es donde aparece claro que la independencia y la distinción no son absolutas; son respectivas a las demás cosas que hay en el Universo. Y esta respectividad de la actividad envuelve el que esa distinción sea no una distinción de mera persistencia pasiva como la de un electrón, sino bien al revés: que sea equilibrio dinámico. Un equilibrio en el que se mantiene la estructura alterada dinámicamente. Y, por tanto, se recobra. Es decir, es una estructura además de dinámica, reversible. En tercer lugar, no solamente es una estructura dinámica y además reversible, sino que entonces, en virtud de esto [...] tiene una cierta independencia del medio, y cierto control sobre él» (Zubiri, 2006, p. 165).

Resumiendo, Zubiri afirma que las estructuras de lo vivo: «son las estructuras de unos sistemas moleculares, que no pueden continuar siendo lo que son más que ejercitando unas actividades en equilibrio diná- 
mico y además reversible, y precisamente teniendo una independencia y un control específico sobre el medio en esa actividad» (Zubiri, 2006, p. 166; Zubiri, 1996 p. 626). En las citas anteriores Zubiri incorpora varias ideas respecto de lo vivo y su comprensión desde la perspectiva físico-química, en las que están implícitos los trabajos de varios científicos durante los años 30 y 40 que concibieron el ser vivo como sistema abierto y homeostático, ideas a las que Prigogine buscó darles una base termodinámica. Veamos los aspectos principales.

Zubiri se refiere al carácter abierto de los sistemas biológicos, a la necesidad de intercambio de energía y materia con el entorno para seguir siendo lo que son, lo que Prigogine denomina su carácter disipativo. El carácter de sistema abierto de los organismos fue destacado por varios autores durante los años 30 y 40. Por ejemplo, ya en 1928 en la revista Nature se afirmaba: "La distinción fundamental entre lo vivo y lo no vivo es que, mientras es posible aislar los fenómenos del mundo inorgánico, es imposible considerar un organismo viviente aparte de su medioambiente» (Editorial, octubre 1928, p. 501). Fue especialmente el trabajo del biólogo teórico austriaco Ludwig von Bertalanffy el que puso de relieve el carácter abierto de los seres vivos. En su artículo de 1940, «Der Organismus als physikalisches System betrachtet» [EI organismo considerado como un sistema físico $]^{4}$ nos dice: «El organismo no es un sistema cerrado sino uno abierto» (Bertalanffy, 1976, p. 125). El carácter abierto implica que hay intercambio de energía y materia con el entorno.

Asociado al carácter de sistema abierto de los seres vivos está el reconocimiento de que la materia viva no se encuentra en un estado de equilibrio en sentido estricto, en un estado de equilibrio estático, sino, tal como afirma Zubiri, en un estado de equilibrio dinámico o en flujo (o estado estacionario [steady state], que es la denominación más extendida). Son los trabajos del fisiólogo inglés $A$. V. Hill, los que a principios de los años 30 ponen de relieve esta característica de lo vivo (Donnan, 1928, p. 514). La diferencia entre el equilibrio estático y el equilibrio dinámico se mienta como una diferencia entre lo vivo y lo muerto. Tanto el estado estacionario como el estado de equilibrio son estados, en el sentido de que mantienen un valor constante en el tiempo. Sin embargo, el estado estacionario requiere un flujo de energía y materia para sostenerse idéntico en el tiempo, mientras que el equilibrio estático, que estudian la termodinámica o la química-física clásicas, no requiere de tal flujo para mantener su condición. En el estado estacionario no se anula la actividad disipativa, los procesos irreversibles y la producción de entropía se mantienen, a diferencia del equilibrio en donde se anulan. Es el dinamismo, la irreversibilidad de los procesos disipativos y el flujo continuo de materia y energía, el que aparece a la base y como sostén de la organización y estructura de los seres vivos. De esta manera, se destaca que la materia viva no se encuentra en equilibrio con su entorno, sino que mantiene un estado de noequilibrio "alimentándose» de la energía libre del medio (Schrödinger, 1985/1944, p. 97).

Zubiri enfatiza también la idea de «distancia» del viviente respecto a su entorno, y su capacidad de sostener o mantener su identidad frente a perturbaciones o «vicisitudes» del entorno. Es lo que denomina su carácter «reversible». Lo implícito aquí es la estabilidad del estado de equilibrio dinámico del ser vivo. El estado de equilibrio dinámico no es sólo una situación mantenida por el flujo de energía y materia, sino que es activamente sostenido por el propio organismo, a través de una serie de coordinaciones y relaciones entre procesos al interior del organismo o sistema vivo, se mantiene el estado estacionario frente a perturbaciones en el medio, se mantiene la estabilidad del sistema. Un ser vivo mantiene de manera activa su condición particular, la fijeza o constancia [fixité] de su «medio interno» [milieu intérieur], respecto al medio externo, como estableció el fisiólogo francés Claude Bernard (1885, pp. 112-113). Este activo sostenimiento de una constancia en flujo es lo que el fisiólogo estadounidense Walter Cannon denominó "homeostasis», inspirándose en la ideas de Bernard (Cannon, 1929, p. 400). La homeostasis se refiere a los procesos que tienen lugar para mantener un estado estacionario determinado, es decir se refiere al carácter autorregulado de los seres vivos (Bertalanffy, 1976, p. 80). Respecto a esto Zubiri afirma: «Sin esto [la homeostasis] el ser vivo no podría mantener sus estructuras. El equilibrio homeostático es algo más que un equilibrio: es un momento dinámico de la actividad de los seres vivos [...] la organización y el equilibrio del medio interno no es un equilibrio estático, es un equilibrio dinámico» (Zubiri, 2006, p. 181). Este carácter auto-regulado implica que los seres vivos son sistemas estables, o al menos metaestables ${ }^{5}$, en el sentido que poseen mecanismos de regulación que les permiten volver a un estado dado frente a perturbaciones o cambios en el ambiente. Los sistemas vivos mantienen su situación frente a perturbaciones o fluctuaciones del medio, a las que son vulnerables por su carácter abierto. La regulación implica que el 
sistema actúa en contra de estas perturbaciones, de tal manera de mantener un estado estacionario o alcanzar uno nuevo. Desde este punto de vista, los seres vivos son sistemas que tienden a conservar lo ya dado. Su actividad tiene un carácter reactivo o conservador, ejerciendo una suerte de control sobre el medio. Antes de proseguir aclaremos un punto para evitar confusiones. Zubiri caracteriza la actividad vital como "reversible», mientras que Prigogine dirá que es «irreversible». Con esto, se refiere al carácter estable de los seres vivos, mientras que Prigogine, siguiendo el uso propio en termodinámica, se refiere al tipo de proceso termodinámico implicado. Un proceso irreversible implica disipación de energía o crecimiento de entropía, mientras que uno reversible no (Durán, 2013, p. 26). Lo que Zubiri mienta con el término "reversible», lo encontraremos en Prigogine en el término «estable».

Ahora bien, Prigogine por su parte, en su primera etapa de trabajo, durante los años 40 (Prigogine, 1947 y 1967; Glansdorff y Prigogine, 1971; Nicolis y Prigogine, 1977; Prigogine y Stengers, 1994), busca establecer los fundamentos termodinámicos de la concepción del ser vivo como sistema abierto y homeostático, entendiendo así el carácter dinámico de los seres vivos como intrínsecamente disipativos, sin implicar por ello una contradicción con la segunda ley de la termodinámica (Durán, 2013). Prigogine busca establecer así una «termodinámica de la vida» (Prigogine, 2004, p. 305), en la que disipación y dinámica vital sean compatibles. Prigogine se ocupa de sistemas abiertos, que intercambian materia o energía con el ambiente, y de estados de no-equilibrio (Prigogine, 1947, p. 2). Este énfasis en los sistemas abiertos y de no-equilibrio, constituye una innovación respecto al enfoque de la termodinámica clásica, centrada en sistemas aislados (o cerrados) y en los estados de equilibrio. El equilibrio constituía el punto final de la actividad espontánea del sistema. Estado en donde se alcanza una homogeneidad global y ya no se producen transformaciones, siendo cero la actividad disipativa. Se trata por tanto de un equilibrio estático. Así, para plantear su termodinámica de procesos irreversibles, Prigogine reformula la segunda ley de la termodinámica ${ }^{6}$, y muestra que en sistemas abiertos son posibles estados estacionarios de no-equilibrio (Prigogine, 1967, pp. 83-4; Bertalanffy, 1949, p. 384), y sobre todo, gracias a la demostración de un principio de mínima producción de entropía (Prigogine, 1945), muestra la estabilidad de los mismos, la capacidad de mantener su identidad, frente a ciertas perturbaciones del medio, lo que Prigogine conside- ra una extensión del principio de moderación de Le Chatelier-Braun a estados de no-equilibrio. Se muestra así la posibilidad de comportamientos dinámicos del mismo tipo que en los seres vivos, en sistemas puramente físico-químicos ${ }^{7}$. Sin embargo, un aspecto queda pendiente en esta formulación. En los sistemas lineales estudiados por Prigogine, el estado estacionario, está determinado por las variables externas. Más que controlar el medio, el sistema es controlado por el medio de manera plena. Su estado interno se define desde el exterior. Este rasgo se verá superado en los sistemas no-lineales estudiados por Prigogine en los años 60 (Nicolis y Prigogine, 1977, p. 46).

Vemos cómo las ideas respecto al carácter dinámico de lo vivo en Zubiri, la concepción de lo vivo como estabilidad o equilibrio dinámico, se asientan en trabajos científicos de los años 30 y 40 , entre los cuales se encuentra el trabajo en termodinámica de procesos irreversibles de Prigogine. La idea principal expresada por estos trabajos es que lo vivo no es equilibrio estático, sino dinámico. El ser vivo se mantiene siendo el mismo de manera dinámica y activa. Ahora bien, esto no implica que el ser vivo sea mero dinamismo, actividad asentada en la nada. También posee estructuras. Es como afirma Zubiri, «sistema de moléculas». El desvelamiento y el énfasis en el carácter estructural del ser vivo se deberá sobre todo al advenimiento de la biología molecular en los años 60 , que renovará por completo la concepción de los seres vivos. Tanto Zubiri como Prigogine incorporarán estas ideas y buscarán compatibilizarlas con la concepción dinámica aquí expuesta.

\section{LOS SERES VIVOS COMO ESTRUCTURA Y DINÁMICA}

La riqueza estructural del ser vivo quedará en evidencia con la eclosión de la biología molecular en los años 60. Así, el término "vida» ya no tiene el mismo sentido que tenía en los años 40, cuando parecía suficiente asentar el carácter abierto y homeostático, el equilibrio dinámico, de los seres vivos. Para la biología molecular, la vida, siguiendo las palabras del biólogo molecular André Lwoff, se entiende principalmente como "orden biológico», orden estructural y funcional: "Un organismo es una unidad integrada de estructura y funciones [...] El orden biológico es dual, estructural y funcional, estático y dinámico [...] El ser vivo -el organismo, la unidad de la vida - ha sido definido como un sistema independiente de estructuras y funciones integradas» (Lwoff, 1962, pp. 8-15) ${ }^{8}$. Vemos expresada aquí la concepción del ser vivo como conjunción de estructura y dinamismo, de 
moléculas y macromoléculas y actividad disipativa. La estructura tiene una connotación espacial, asociada principalmente con la disposición de las partes que constituyen moléculas o macromoléculas. El ejemplo característico es la estructura de doble hélice del ADN. Por su parte, lo funcional se refiere a los procesos y relaciones temporales entre distintos componentes y estructuras (procesos como metabolismo, crecimiento, reproducción) (Monod, 1985, p. 68), que implican un dinamismo disipativo. Se conjuga así lo espacial y lo temporal ${ }^{9}$.

Ahora bien, estos dos momentos de lo vivo, lo estructural y lo dinámico, no están en un mismo pie: lo funcional o dinámico está determinado por lo estructural. Existe así una prioridad o primacía de lo estructural respecto de lo funcional, de lo espacial respecto de lo temporal. Esta prioridad está implícita ya en la comprensión de lo vivo como "orden biológico» ${ }^{10}$, y podemos decir que aquí orden es sinónimo de estructura, en cuanto disposición espacial ya dada. En sentido preeminente, orden biológico significa orden estructural o espacial. Justamente en la interpretación de esta prioridad, de este antes, yacerá la distinta comprensión de lo vivo y su dinámica particular. Para una línea de pensamiento preponderante en la biología molecular, la prioridad no es sólo lógica o formal, sino temporal. En este sentido, lo estructural (sobre todo la estructura del ADN), está completa ya antes de su «desenvolvimiento» temporal (Monod, 1985, p. 107). Sustancias como el ADN pueden llamarse vida en sentido propio, pues contienen el plan o programa (Jacob, 1973, p. 9) de la actividad vital. Esta posición puede asociarse a la concepción de «orden a partir del orden» de Schrödinger (1944/1985, p. 108), o también al llamado "determinismo genético» (Keller, 2000 , p. 21). Lo temporal no agrega nada que ya no estuviera en la estructura espacial. Desde otro punto de vista, la prioridad de lo estructural sobre lo funcional, se entiende sólo desde una perspectiva formal: las estructuras determinan las actividades del viviente en el dinamismo vital mismo. Lo estructural es distinguible, pero no separable de lo dinámico, y por tanto, sustancias como el ADN, no son ni están vivas, sino que juegan un rol determinando y dirigiendo el conjunto de funciones que permite la creación de nuevas estructuras y la mantención de la identidad y unidad del ser vivo a través del tiempo (desarrollo, metabolismo, reproducción, evolución). Esta es la posición, por ejemplo, de Lwoff: «El organismo viviente es un sistema integrado de estructuras macromoleculares y funciones disponibles para reproducir su especie [...] Separada de su contexto - esto es, extraída de la célu- la - cualquier estructura, sea un ácido nucleico o una proteína, es sólo una molécula orgánica. Tal cosa como una sustancia viva o materia viviente no existe. La vida sólo puede ser la propiedad del organismo como un todo. Sólo los organismos están vivos» (Lwoff, 1962, p. 100). Mencionemos, por último, la posición que aceptando la comprensión del ser vivo como estructura y dinámica propia de la biología molecular, se opone a ésta en cuanto enfatiza el proceso antes que la estructura. Esta es la posición por ejemplo del embriólogo austriaco Paul Weiss, para quien: «El proceso dinámico es el fundamento de la forma estática, más que al revés» (Weiss, 1968, p. 25). Prigogine también enfatizará el proceso respecto a la estructura, afirmando: «Es la función la que crea la estructura» (Prigogine, 2006, p. 34). Sin embargo, las propias herramientas teóricas que usa implican más bien lo contrario, como veremos a continuación.

A mediados de los años 60, Prigogine entra en una segunda etapa de su trabajo, centrándose en el estudio de sistemas físico-químicos no-lineales lejos del equilibrio (Prigogine, 1967; Glansdorff y Prigogine, 1971; Nicolis y Prigogine, 1977; Prigogine y Stengers, 1984 y 1994). Encuentra que bajo ciertas condiciones, las situaciones de inestabilidad adquieren una dimensión creativa ${ }^{11}$, conduciendo a la aparición espontánea de ordenaciones o regularidades espaciales o temporales, que requieren energía o materia para sostenerse (Prigogine, 1967, p. 117 y 1969, p. 23). Un ejemplo experimental clave es el de Bénard. Sistema hidrodinámico en el que dada cierta distancia del equilibrio se produce una inestabilidad que da lugar a la aparición espontánea de movimientos convecti$\operatorname{vos}^{12}$. O el experimento de Belousov-Zhabotinsky en el ámbito de la química, en el que se produce la aparición de diversos patrones espaciales y temporales (Prigogine y Nicolis, 1971a). Prigogine denomina «estructuras disipativas» a las "formas o patrones» que aparecen en este tipo de sistemas, tratando de aunar la dimensión estructural y dinámica (o disipativa) que implican (Prigogine y Stengers, 1994, p. 181). Se trata de "estructuras» dinámicas estables, que requieren de un continuo intercambio para mantener su identidad, a diferencia de las "estructuras» de equilibrio, como los cristales, que no requieren de este intercambio para mantenerse siendo lo que son (Prigogine, 1969, pp. 23-24). Prigogine denomina autoorganización al proceso que da lugar a estas estructuras disipativas, mostrando así claramente su intención de establecer su teoría como una clave en la comprensión de los seres vivos desde una perspectiva material (Prigogine, 2004, p. 161; p. 175). Con su teoría de es- 
tructuras disipativas y procesos de autoorganización, Prigogine intentará comprender lo vivo en su doble dimensión estructural y dinámica (Prigogine, 1972, p. 305; Prigogine, 1977, pp. 255-256; Prigogine, 1969, p. 50; Prigogine, 1971, p. 181) $)^{13}$. Al mostrar la posibilidad de autoorganización en sistemas puramente físicoquímicos, Prigogine afirma la disipación como fuente de vida, rompiendo con la imagen contradictoria que ésta tenía en la termodinámica clásica. La existencia de procesos organizados en el espacio y en el tiempo, no se contradice con la producción de entropía o la disipación, por el contrario, en cuanto procesos de equilibrio dinámico, la requieren para su conservación y estabilidad ${ }^{14}$. Prigogine pretende así asentar el valor creativo de la irreversibilidad, establecer científicamente lo que Bergson denominaba «tiempo creador», por tanto la creatividad del dinamismo, de lo procesual. Desde esta perspectiva, su énfasis es en el carácter dinámico de lo vivo, en la aparición de "estructuras» a partir de procesos disipativos. No obstante, la disipación o irreversibilidad no es el único ingrediente necesario para la autoorganización. Se necesitan además relaciones no-lineales y auto-catálisis, además de fluctuaciones ${ }^{15}$. Se requiere así de una estructura u organización que define la identidad o unidad del sistema. Respecto a esto tenemos que considerar algunos puntos clave.

Primero, lo que Prigogine denomina "estructura» se refiere sólo a ordenaciones o regularidades espaciales o temporales. Se refiere sólo a «patrones o formas», no a relaciones constitutivas de un sistema. Segundo, las estructuras disipativas son un estado de un sistema, no el sistema mismo. Tercero, este sistema está definido por un esquema o modelo de reacciones químicas ${ }^{16}$, que establece una unidad funcional o sistémica clausurada, estableciendo las relaciones entre cada uno de los elementos que componen el sistema. Cuarto, el esquema es a-temporal, pero determina la dinámica temporal del sistema, expresada por las ecuaciones diferenciales de sus componentes ${ }^{17}$. Quinto, el esquema de reacciones constituye la unidad e identidad del sistema, propiamente hablando su estructura u organización. Sexto, este esquema establece las relaciones no-lineales y de auto-catálisis que permiten la inestabilidad y las múltiples opciones o estados estables disponibles. Séptimo, la unidad de este esquema no puede ser expresada como suma de reacciones, o sumatoria de efectos. En síntesis, los sistemas que estudia Prigogine no implican creación o aparición de ordenaciones o "estructuras» a partir de un mero dinamismo, como afirma él mismo ${ }^{18}$, sino que los procesos de autoorganización implican la concreción aleatoria o espontánea de estados o soluciones establecidas de antemano por la estructura $u$ organización del sistema (el esquema de reacciones). Así, el esquema de reacciones define lo que el sistema es, y lo que puede ser, no como una realización en el tiempo, sino como un momento de la propia estructura. De esta manera, lo que Prigogine denomina "estructura» disipativa no es producto de un puro dinamismo, irreversibilidad o proceso, sino que nace de un dinamismo ya estructurado u organizado.

Zubiri, por su parte, en su concepción de lo vivo como estructura dinámica, se hará eco de los desarrollos de la biología molecular, tomando una posición en cierta medida equivalente a la de Lwoff, arriba mencionada, en cuanto considera lo vivo en su unidad de estructura y dinámica, con énfasis en la primera. Se da una primacía de lo estructural, que no es nada aparte del dinamismo, sino un momento de la propia ejecución de las actividades del viviente. Zubiri lleva, sin embargo, un paso más allá este "estructuralismo», al concebir lo dinámico como un momento no esencialmente temporal, sino estructural. Distingue así, sin separar, lo dinámico de lo temporal (Zubiri, 2006, p. 279; Zubiri, 1996, p. 261). Recodemos que para Zubiri las cosas reales son sustantividades o sistemas de notas respectivas (notas-de) clausurados y concatenados (cada nota tiene una posición dentro del sistema) (Zubiri, 2006, p. 31; Zubiri, 1998, p. 144; Zubiri, 2001, p. 41; Zubiri, 1996, p. 400). Desde la perspectiva de la unidad del sistema, tenemos un constructo (fundado en última instancia en la unidad de lo que Zubiri denomina esencia (Zubiri, 1998, p. 143; Zubiri, 2001, pp. 60-61 $)^{19}$, mientras que desde la perspectiva de las notas, esta unidad es un ex-tructo, una estructura (Zubiri, 2006, p. 37). Así, lo que Zubiri concibe como estructura se acerca más a lo que antes encontramos en Prigogine asociado al esquema de reacciones. Para Zubiri esta estructura "da de sí», no como algo consecutivo o posterior, sino de manera constitutiva, en cuanto es "activa por sí misma» (Zubiri, 2006, p. 61; Espinoza, 2006, p. 49). Al distinguir dinamismo de tiempo, Zubiri incorpora el «dar de sí» como un momento estructural, en un sentido muy cercano al implícito en los sistemas estudiados por Prigogine, en cuanto el sistema, definido por su estructura u organización, no es sólo un estado temporal presente, sino que todo lo que puede dar (en este caso los estados que puede ocupar) (Durán, 2013, p. 159). Esta distinción entre dinamismo y tiempo, está en línea con el intento de Zubiri de pensar el devenir sin apelar al no-ser, no-ser que se infiltra cuando se concibe el dinamismo como cambio o desde la perspectiva del ser (Zubiri, 2006, p. 11). 
Como es habitual, Zubiri posiciona su concepción de lo vivo como estructura dinámica, en contra de posiciones tradicionales. Rechaza la vida concebida como sustancia, y la vida concebida como puro dinamismo. "Una estructura viva, en primer lugar no es una estructura sustancial. Los seres vivos, los organismos, no son sustancias: son estructuras. Estructuras materiales, ni que decir tiene, pero son estructuras. Lo característico de la vida es una estructuralidad y no una sustancia. Sustancias, hay millones en cada ser vivo, aun en la ameba más elemental. Un ser vivo no es eso. Un ser vivo no es una sustancia más, ni tan siquiera una super-sustancia: es una estructura. En segundo lugar, tampoco se puede decir que la vida sea un mero dinamismo [...] Si la vida no tuviese unas estructuras básicas, no habría proceso en cuestión. En tercer lugar, la cuestión está en determinar cuáles son esas estructuras y cómo de ellas emerge algo que aun siendo un proceso, sin embargo no es formalmente procesual. Por esto decía que no es una estructura sustancial, ni es un mero dinamismo, sino que es un dinamismo estructural: es una estructura dinámica» (Zubiri, 2006, p. 167).

El rechazo de la vida como sustancia tiene dos aspectos principales. Por un lado, hace referencia al rechazo de la concepción aristotélica del cosmos como taxis de sustancias (Zubiri, 2006, p. 42), que implica un dualismo entre cosa y acción. Esta crítica a la concepción sustancialista es una característica fundamental y recurrente del pensamiento de Zubiri, y la enfoca desde diversos ángulos. No es, sin embargo, una crítica exclusiva suya, y la podemos encontrar, por ejemplo, en autores como Whitehead (Leclerc, 1956). Por otra parte, la crítica a la vida como sustancia, se refiere al rechazo de considerar una sustancia particular como vida o como algo vivo. "El ser vivo no es una sustancia sino millones de sustancias. Y entonces, propiamente hablando, el ser vivo es una estructura; no es una sustancia» (Zubiri, 2006, p. 190). El ADN fuera de la sustantividad viva es sólo una molécula orgánica más o incluso menos, es una mera "cosa material» (Zubiri, 1996, p. 388). Esta posición de Zubiri es similar a la de Lwoff, citada más arriba. En el ámbito del desarrollo embrionario, el rechazo de la vida como sustancia, afirma también que la organización o desarrollo vital no es producto de sustancias "organizadoras», que gracias a sus propiedades químicas particulares ejercerían un rol director (Zubiri, 2006, p. 190). Posición contra la cual también se pronuncia Prigogine (Prigogine y Stengers, 1984, p. 174; Stengers, 1985, p. 74) $)^{20}$. Así, lo vivo no está caracterizado esencialmente por su materia, por sus componentes o sustancias, sino por su estructura sistémica, por su organización. En este sentido, y manteniendo las importantes diferencias entre ellos, Zubiri entra en la línea de autores como Weiss, Bertalanffy (1963), Maturana y Varela (1994), y el propio Prigogine, quienes, cada uno a su modo, han buscado comprender la vida desde el conjunto de relaciones que implica, más que desde la materia específica de que están compuestos los seres vivos.

Un segundo punto, puesto de relieve en la cita, es el rechazo de la concepción de lo vivo como puro dinamismo. Para Zubiri no hay proceso sin estructura, y su rechazo al ser vivo como dinamismo puro (Zubiri, 2006, p. 167) se refiere sobre todo a Bergson, cuya figura está muy presente en Estructura dinámica de la realidad. Para Bergson, el élan vital es puro dinamismo, pura vitalidad, sin mezcla de materialidad, y las virtualidades que lo constituyen no pueden considerarse una estructura (Bergson, 2007, p. 263; p. 252 ; p. 103). Aunque la organización de lo vivo implica una conjunción de vida y materia, un modus vivendi de ambas, la vitalidad en cuanto tal es inmaterial (Bergson, 2007, p. 256). En otro pasaje de Estructura dinámica de la realidad, Zubiri hace explícito el rechazo al «vitalismo» de Bergson: «¿Qué es el soplo de la vida si no es el ejercicio de las actividades que derivan de unas estructuras y que revierten sobre ellas?» (Zubiri, 2006, p. 186). Con esto no niega, sin embargo, el carácter creador de la vida, puesto de manifiesto con tanta fuerza por Bergson, y que Prigogine creerá encontrar en las inestabilidades que dan lugar a procesos de autoorganización. La vida tiene para Zubiri una dimensión de creación que no consiste, no obstante, en "un élan "inventor" "(Zubiri, 2006, p. 186). No hay un puro dinamismo creador, sino estructuras dinámicas.

De esta manera, para Zubiri la vida no está montada en la nada, sino que sobre estructuras y dinamismos previos (Zubiri, 2006, p. 176). Esta concepción jerárquica de lo vivo la encontramos expresada, por ejemplo, en la «auto-organización» del embriólogo austriaco Paul Weiss (1968), concepción que influirá en Prigogine (Durán, 2013, p. 253). Weiss expresa la jerarquía de organizaciones de lo vivo con la expresión "organización a partir de lo organizado». «El principio de continuidad sin quiebres de la organización de los sistemas vivos, que una vez expresé como 'omnis organisatio ex organisatione', con la comprensión de que grados más altos de organización pueden emerger de la libre interacción de sistemas organizados y predispuestos de orden de complejidad más bajo» (Weiss, 1968, p. 130). Weiss (1968) concibe la organi- 
zación, comprendida también desde una perspectiva estructural o sistémica (cf. p. 96), como emergiendo de organizaciones previas, aunque no siendo reducible a ellas. Zubiri reconoce la jerarquía existente entre los dinamismos que describe, y la necesidad de estructuras previas para el sostenimiento de otras más complejas (Zubiri, 2006, p. 176, p. 181).

Como vemos, tanto Zubiri como Prigogine, con sus concepciones de lo vivo como "estructura dinámica» y «estructura disipativa», respectivamente, intentan conjugar la dimensión estructural y dinámica del ser vivo, puestas de relieve por los desarrollos de la biología molecular y el estudio de los sistemas abiertos y homeostáticos. El ser vivo como unidad estructural y dinámica aparece como algo incuestionable. La cuestión está en cómo concebir esta unidad. Para Prigogine, siguiendo a Bergson y Weiss, es el proceso dinámico el que da lugar a la "estructura». Entendiendo estructura, sin embargo, en sentido de mero "patrón o forma» espacial o temporal. Pierde de vista así, que las "estructuras» disipativas nacen de un proceso ya organizado o estructurado. Zubiri, por su parte, enfatiza el momento estructural, a tal punto que concibe el dinamismo como un momento de la estructura, como distinto del tiempo. En ambas propuestas nos encontramos con una mismidad, con un sí mismo, que constituye la unidad de los momentos estructural y dinámico. Tenemos que analizar en qué medida el "sí mismo» de la dinámica de la mismidad es equivalente al «sí mismo» de la auto-organización, y si en ésta se da el dinamismo propio de lo vivo, tal como lo entiende Zubiri.

\section{MISMIDAD, SÍ MISMO Y AUTO-ORGANIZACIÓN}

Zubiri distingue dos momentos en el dinamismo de la mismidad, que apuntan a la unidad de dinámica y estructura implícita en ella (Zubiri, 2006, p. 192). El primer momento, que podemos llamar de talidad, se refiere a la unidad estructural de los procesos dinámicos que mantienen la identidad y estabilidad del ser vivo, el equilibrio dinámico estructural del que hemos hablado más arriba. El "sí mismo» desde esta perspectiva consiste justamente en las estructuras que se mantienen siendo tales en y por las actividades del viviente. El segundo momento se refiere a la realidad en cuanto tal, lo que Zubiri en esta época denomina la «forma o modo de realidad» que implica el ser vivo por su dinamismo particular (Zubiri, 2006, p. 184). Zubiri dirá que el "sí mismo» desde esta perspectiva consiste en un "poseerse»: "Sí mismo es poseerse» (Zubiri, 2006, p. 198)
El "sí mismo» desde la perspectiva dinámica consiste en ser el mismo sin ser lo mismo: «El viviente es siempre el mismo pero nunca lo mismo» (Zubiri, 2006, p. 200; p. 199). Las estructuras del viviente, su unidad estructural, es la misma, nunca siendo el viviente lo mismo. Las estructuras son previas a la mismidad dinámica, pero no aparte de ella, sino en y por ella. «La mismidad es consecutiva a las estructuras. Es consecutiva al autós, no es anterior al él» (Zubiri, 2006, p. 193). El sí mismo consiste en las estructuras que dan identidad al viviente, pero que no son tales, ni pueden sostenerse sin su momento dinámico. Existe una anterioridad de la identidad en la propia actividad. Es ese "ya» el que Zubiri enfatiza con lo que denomina «reduplicación»: "La mismidad no es una mera persistencia sino que es el acto reduplicativo y formal en que un ser vivo ejecuta unas acciones precisamente para ser aquello que estructuralmente ya era» (Zubiri, 2006, p. 185). La mismidad del ser vivo contrasta así con la mera permanencia pasiva de un electrón (ejemplo favorito de Zubiri) (Zubiri, 2006, p. 185), pero también con la permanencia de las «estructuras de equilibrio» como los cristales, tal como destaca Prigogine, pues no requieren energía o materia para mantener su identidad. El ser vivo, por el contrario, permanece en estado de equilibrio dinámico, ejercitando sus actividades "para poder ser el mismo que era antes» (Zubiri, 2006, p. 185).

Esta concepción de la mismidad dinámica del viviente, le permite a Zubiri rechazar otras concepciones tradicionales del "sí mismo». En primer lugar, que el sí mismo propio de la vida sea un sujeto o una sustancia (Zubiri, 2006, pp. 189-90). La vida, como ya hemos visto, no es sustancia, sino que es estructura. Estructura que tiene una unidad interna: "cuya plasmación en el sistema constructo de notas es justamente lo que constituye toda la riqueza estructural del ser vivo. Esta unidad estructural no es un sujeto del cual broten precisamente las notas estructurales, sino que es algo interno y además intrínseco al sistema mismo, en cuyas notas está presente, de una manera dominante y exigente» (Zubiri, 2006, p. 190). Zubiri rechaza también que se trate de mera identidad (identidad formal $A=A$ ) o de identidad lograda, resultado o logro de la vida, de las actividades vitales, tal como habría concebido el "sí mismo» el idealismo alemán (Zubiri, 2006, p. 191). El sí mismo no puede ser mera identidad formal, ni algo consecutivo a las actividades, sino que está antes y en su propia ejecución. "La vida es el movimiento de un algo que estructuralmente es "sí mismo" y que precisamente por serlo puede conferir ese carácter de 
mismidad, y ese carácter de quiescencia a los movimientos vitales. El sí mismo, el autós, no es un resultado de la vida sino un principio de ella» (Zubiri, 2006, p. 192). Así, la unidad estructural, el «sí mismo» del ser vivo desde la perspectiva de la dinámica, no es sujeto ni mera identidad, «sino que consiste en algo mucho más estricto y riguroso: en ser siempre el mismo» (Zubiri, 2006, p. 193), el mismo que ya era por poseer sus estructuras.

Desde una perspectiva más radical, desde el punto de vista del momento de realidad, el "sí mismo» no consiste en ser mera mismidad dinámica: «La mismidad es esencial y formalmente un acto de poseerse» (Zubiri, 2006, p. 185). El sí mismo, el de suyo propio de lo vivo es poseerse. "Lo que desde el punto de vista del dinamismo es el dinamismo de la mismidad, desde el punto de vista radical, y como forma de realidad, la vida es justamente un poseerse» (Zubiri, 2006, p. 185). Este poseerse, implica que el "sí mismo» es intrínseco al viviente, que en la ejecución de sus actividades las estructuras que lo constituyen se poseen a sí mismas (Zubiri, 2006, p. 194). Ahora bien: "¿̇en qué consiste esta pertenencia intrínseca del dinamismo vital, del dinamismo de la mismidad, al viviente en cuanto tal?» (Zubiri, 2006, p. 194).

Para responder a esta pregunta, Zubiri parte nuevamente rechazando posiciones clásicas, no consideradas falsas por completo, sino insuficientes. En primer lugar, rechaza que la pertenencia sea espontaneidad, aspecto que será de vital importancia al comparar estas ideas con las de Prigogine. Según esta posición, las actividades del viviente le pertenecen en cuanto nacen de él de manera absolutamente espontánea (Zubiri, 2006, p. 187; p. 194). No obstante, nunca encontramos esta espontaneidad absoluta, sino a lo más una espontaneidad relativa. En primer lugar, porque los seres vivos están inmersos en un medio, que los fuerza siempre a actuar (Zubiri, 2006, p. 195). En segundo lugar, tiene un medio interno que lo fuerza (Zubiri, 2006, p. 195), y por último, cada parte que lo constituye actúa sobre la otra. "Los momentos estructurales de un ser viviente actúan los unos sobre los otros, y por eso el viviente produce sus acciones» (Zubiri, 2006, p. 195). Así, no encontramos nada que actúe de manera absolutamente espontánea o libre en el viviente. Pero sobre todo, Zubiri rechaza la espontaneidad en cuanto implica un dualismo de viviente y actividad, pues implica que el viviente es ya tal antes de su dinamismo. La espontaneidad implica que el viviente se activa por sí mismo, no que consista en actividad misma (Zubiri, 2006, p. 197).
Prigogine por su parte, considerará la espontaneidad con que aparecen las "estructuras disipativas» una característica clave para comprender lo vivo, y es a esta característica a la que apunta cuando habla de «autoorganización»: "Autoorganización, la aparición espontánea de una actividad diferenciada en el tiempo y en el espacio" (Prigogine, 2004, pp. 92-3). ¿Qué se entiende aquí por espontaneidad? En primer lugar, hay que tener en cuenta que los sistemas termodinámicos siempre son espontáneos, en el sentido que actúan "por sí mismos». Por ejemplo, un sistema aislado se conduce por sí mismo hacia un estado de equilibrio. Sin embargo, este estado es controlable desde el exterior del sistema (a través de las condiciones de frontera), variando el flujo de energía o materia, o en otros términos la «distancia del equilibrio» (Durán, 2013, p. 161). En el caso de las inestabilidades que conllevan la aparición de «estructuras» disipativas, estas condiciones no controlan el estado futuro (Durán, 2013, p. 233 y p. 26; Stengers, 2003, p. 227). Esto se debe a que la inestabilidad implica múltiples opciones, concretándose de manera aleatoria sólo una, debido a fluctuaciones o perturbaciones. En un primer momento, Prigogine asoció la autoorganización a fluctuaciones internas, buscando otorgar así una espontaneidad absoluta al sistema (Prigogine y Nicolis, 1971b; Durán, 2013, p. 209). No obstante, tuvo que abandonar esta posición, pues resulta mucho más probable que el proceso sea gatillado por una perturbación externa (Durán, 2013, p. 226). Como vemos la espontaneidad de la autoorganización no es absoluta, y termina siendo entendida como aleatoriedad, ya sea interna o externa, como elemento no controlable por quien define el sistema. Los procesos de autoorganización, tal como los estudia Prigogine, son activos en sí mismos, pero no por sí mismos, porque requieren una activación externa.

Zubiri rechaza, por otra parte, que el poseerse del viviente sea inmanencia, que el dinamismo le pertenezca al ser vivo simplemente por permanecer siendo lo que es, por ser persistencia meramente pasiva (Zubiri, 2006, p. 187; p. 188; p. 196). Rechaza igualmente que la pertenencia sea emergencia o "acción inmanente» (Zubiri, 2006, p. 195). En definitiva, ni la inmanencia, ni la emergencia, son diferentes a la espontaneidad, en cuanto sigue implícito en ellas un dualismo del viviente y sus actividades, o de las estructuras y el dinamismo, y esta es la razón principal para rechazarlas como caracterización del sí mismo en cuanto poseerse (Zubiri, 2006, p. 197). «No se trata de cómo pertenece el movimiento vivo al viviente, 
ni de cómo surge el movimiento vital en el viviente, sino de cómo el viviente pertenece al movimiento. No de cómo el movimiento pertenece al viviente. El viviente es un "sí" mismo. $Y$ en el dinamismo de la vida entran formalmente sus estructuras, de manera tal que es en la mismidad de éstas donde las estructuras son plenamente lo que son. El movimiento de la vitalidad, el movimiento de la mismidad, no se limita a salir del viviente y a quedar en él, sino que en él y por él es como siguen siendo "mismas" las estructuras, unas estructuras que son "mismas". Vivir no es sino esto: se reduplicativamente y formalmente sí mismo, es decir, ser estructuralmente el mismo. Las estructuras están en el movimiento [...] las estructuras estarían "para" algo completamente determinado: para ser justamente "mismas", tales y como son. Por esto, en la medida precisamente en que están las estructuras en el movimiento vital para poder ser igualmente aquello que son, por eso es por lo que digo que el dinamismo de la vitalidad pertenece al viviente, y el viviente pertenece al dinamismo de la vitalidad, en esa forma concreta que yo digo "poseerse" [...] [Poseerse] consiste en ejecutar formalmente su mismidad» (Zubiri, 2006, p. 197).

De esta manera, el dinamismo le pertenece al viviente no como algo posterior a sí mismo, sino en cuanto en la ejecución de éste el ser vivo es el mismo que ya era, y sólo puede serlo en la ejecución de sus actividades vitales, de su dinamismo. La unidad estructural está en las actividades que ejecuta el viviente, aunque no se reduce a ellas, sino que les otorga el carácter de mismidad, de "de suyo». Zubiri destaca dos aspectos del "sí mismo" de los seres vivos. Son mismos en cuanto conservan su mismidad dinámicamente, y se poseen a sí mismos en y por este dinamismo. Considerando a una los dos momentos de talidad y realidad del dinamismo propio del viviente, tenemos que: «La vida consiste en ser el mismo y poseerse» (Zubiri, 2006, p. 199; p. 188).

Ahora bien, ¿podemos decir que en los sistemas estudiados por Prigogine, no sólo encontramos una "mismidad» de equilibrio dinámico en las «estructuras» disipativas, sino que además la autoorganización implica un "sí mismo» que sólo es tal en y por su actividad dinámica? Para que los sistemas estudiados por Prigogine coincidieran con lo propio de lo vivo, tal como lo describe Zubiri, tendríamos que encontrar en ellos un "sí mismo" que fuera tal sólo en la ejecución de las actividades que determina. Para investigar esto es necesario recordar una distinción ya destacada: la «estructura» disipativa es sólo un estado del sistema que se auto-organiza, no es el sistema mismo. El mismo sistema puede estar en equilibrio estático, en estado estacionario homogéneo, en un umbral de inestabilidad, o en una estructura disipativa. Dependerá de las condiciones externas o de control que se le apliquen (Durán, 2013, p. 161). El «sí mismo» del sistema está dado por el esquema de reacciones, su estructura u organización. Éste constituye un modelo a-temporal que define los estados posibles del sistema, y del cual se obtienen las ecuaciones diferenciales que establecen la actividad del mismo en el tiempo (Durán, 2013, p. 159). La unidad de este esquema es la que está siempre implícita en cada uno de los estados del sistema. Tenemos así dos ámbitos de «mismidad»: la «mismidad» de la «estructura» disipativa, en cuanto estado estable del sistema, y la mismidad del sistema mismo. La «mismidad» de la primera consiste en la permanencia en el tiempo de las ordenaciones o patrones espaciales o temporales en que consiste. Esta identidad se sostiene sólo gracias a procesos disipativos, y a la existencia de una mismidad estructural. Las "estructuras» disipativas no son activas por sí mismas, sino el resultado de la actividad del sistema. La analogía no se establece entre el sistema biológico y la «estructura» disipativa, como quiere Prigogine, sino entre el primero y el sistema autoorganizado en su conjunto. Tenemos que preguntarnos entonces si la mismidad de este sistema necesita de las actividades que determina para seguir manteniendo su identidad.

Tengamos en cuenta, primero, que los sistemas que estudia Prigogine no se auto-organizan en sentido estricto. Cuando hablamos de auto-organización implicamos que un sistema por su propia actividad se da a sí mismo su organización e identidad (unidad y clausura) (Durán, 2013, p. 232). Los sistemas físico-químicos estudiados por Prigogine no cumplen esta condición. La organización del sistema, dada por el esquema de reacciones, no es producto de la propia actividad del sistema, sino que está puesta de antemano, por quien lo define. El sistema tampoco se da a sí mismo su límite espacial, que se define de manera independiente al esquema reacciones (Durán, 2013, p. 233). Así pues, ni la clausura organizacional, ni la clausura espacial se deben a la propia actividad del sistema. La unidad y las relaciones que lo definen no nacen de su propia actividad, ni requieren de ella para ser lo que son. De esta manera, la estructura de los sistemas estudiados por Prigogine es anterior, formal y temporalmente a su actividad o dinamismo. El 
"sí mismo» determina las actividades temporales, expresadas por las ecuaciones diferenciales, pero no requiere de éstas para ser lo que es. Determina los estados posibles y las actividades del sistema en el tiempo, pero no es determinado por ellas. No requiere de ellas para mantener su identidad. El sistema no actúa para conservar su mismidad, sino que actúa para "ocupar» un estado. Por otra parte, la estabilidad del sistema no coincide con la estabilidad de sus estados. Así el equilibrio dinámico de las "estructuras» disipativas no es extensible al sistema en su conjunto. Su estabilidad entra en lo que se denomina "estabilidad estructural», la estabilidad de la propia estructura del sistema (Nicolis y Prigogine, 1977, p. 68; Durán, 2013, p. 223 y p. 309). Si bien Prigogine estudia algunos modelos de inestabilidad estructural en relación a cambios evolutivos (Nicolis y Prigogine, 1977, p. 438 y p. 459), su estudio de procesos de autoorganización parte de esquemas de reacciones que no cambian en el tiempo. Están fijos por definición.

Los sistemas "autoorganizados» de Prigogine son «mismos» en cuanto definidos por un esquema de reacciones que determina sus actividades, pero este esquema no requiere de éstas para el ser «el mismo». Su mismidad estructural no pende del ejercicio de las actividades que determina. El equilibrio dinámico, por otra parte, sólo es aplicable a estados del sistema, no al sistema como un todo. De esta manera, los sistemas estudiados por Prigogine no cumplen las condiciones de mismidad de lo vivo establecidas por Zubiri, no se poseen. Las estructuras de los sistemas "autoorganizados» no son estructuras vivas, tal como las entiende Zubiri, pues éstas «se llaman vivas en la medida precisamente en que estructuralmente pueden dar, y tienen que dar dinámicamente unas acciones en las cuales, y sólo en las cuales estas estructuras están poseyéndose» (Zubiri, 2006, p. 200). Los sistemas estudiados por Prigogine, por último, implican así todavía un dualismo de estructura y actividad, que Zubiri intenta superar con su noción de «estructura dinámica».

\section{CONCLUSIONES}

Hemos revisado de manera comparada las propuestas de Zubiri y Prigogine con las que intentan concebir el ser vivo como unidad de estructura y dinámica, sus concepciones de estructura dinámica y estructura disipativa en procesos de autoorganización, considerando además el contexto científico en el que se elaboraron sus propuestas. Como principales aspectos comunes tenemos: a) la concepción de lo vivo como sistema abierto en equilibrio dinámico; b) la aceptación de la dimensión estructural del ser vivo, puesta de relieve por la biología molecular; c) la búsqueda de una comprensión de lo vivo que toma en cuenta el ámbito de sus relaciones constitutivas, por sobre el tipo de materiales de que están compuestos lo seres vivos. Se encuentra en ambos un rechazo de la vida entendida como sustancia. Como principales diferencias: a) lo que Prigogine denomina "estructura» no equivale a lo que Zubiri considera como tal, que apunta más bien al esquema de reacciones que dotan de unidad e identidad a los sistemas estudiados autoorganizados; b) Zubiri enfatiza el momento estructural más que el dinámico, mientras Prigogine hace justo lo contrario, aunque sus herramientas teóricas implican una posición «estructuralista» aún más enfática que la de Zubiri; c) Los sistemas estudiados por Prigogine no son vivos desde la perspectiva de Zubiri, en cuanto su "sí mismo» no necesita de las actividades que determina para ser tal; d) En Prigogine encontramos aún un dualismo de estructura y dinámica, que Zubiri en cierta medida supera con su concepción de estructura dinámica; d) Zubiri no incorpora la inestabilidad y su papel creativo, tal como está de manifiesto en Prigogine, y que en su dimensión de «inestabilidad estructural» podría relacionarse con el estudio de la evolución, y el dinamismo de alteridad.

\section{AGRADECIMIENTOS}

En la elaboración de este artículo R. Durán recibió financiamiento de la Pontificia Universidad Católica de Valparaíso, a través de un proyecto interno de investigación Postdoctoral.

\section{NOTAS}

1. Este artículo es parte del Proyecto Realidad y técnica en Zubiri (Fondecyt $N^{\circ}$ : 1140973).

2. Respecto al "estatuto epistemológico» de los textos de esta época cf. Gracia (1986, p. 203).

3. Respecto a la noción de vida en Zubiri cf. Vargas (2009).
4. Este artículo corresponde al capítulo 5 de Bertalanffy (1976), del cual citamos.

5. La estabilidad a secas implica regulación frente a cualquier tipo de perturbación. La metaestabilidad, estabilidad respecto a cierto tipo de perturbaciones, pero no todas.
6. Plantea la función «producción de entropía» como una propiedad intrínseca de los sistemas, y la «variación de entropía» como un balance entre la producción de entropía y el flujo de entropía con el ambiente: En donde (Prigogine, 1947, p. 2).

7. Incluso Prigogine llega a ofrecer un ejemplo físico: la termodifusión o el 
efecto Knudsen (Prigogine, 1967, pp. 65-66; véase también Glansdorff y Prigogine, 1971, p. 35).

8. «La dualidad entre aspectos estructurales y funcionales es realmente la propiedad esencial del orden biológico» (Prigogine y Lefever, 1975, p. 25).

9. Respecto a los diversos aspectos de la temporalidad biológica cf. Vargas y Espinoza, 2013; Espinoza, 2006, p. 101.

10. «Orden puede ser considerado como una secuencia o sucesión en el espacio o en el tiempo» (Lwoff, 1962, p. 9).

11. Una influencia importante en esta reconceptualización de la inestabilidad son los modelos matemáticos de la morfogénesis del matemático inglés Alan Turing (Durán, 2013, p. 155).

12. Circulación de materia, en este caso líquido, dada la aplicación de un gradiente o diferencia de temperatura. En el ejem-

\section{BIBLIOGRAFÍA}

Bergson, H. (2007). La evolución creadora. Buenos Aires: Cactus.

Bernard, C. (1885). Leçons sur les phénomènes de la vie communs aux animaux et végétaux (vol. I). Paris: J.-B. Bailliere et fils.

Bertalanffy, L. von (1949). Open Systems in Physics and Biology. $\mathrm{Na}$ ture, 163, 12, p. 384. http://dx.doi. org/10.1038/163384a0

Bertalanffy, L. von (1963). La concepción biológica del cosmos. Santiago de Chile: Universidad de Chile.

Bertalanffy, L. von (1976). Teoría general de los sistemas. México D. F.: Fondo de Cultura Económica.

Cannon, W. B. (1929). Organization for Physiological Homeostasis. Physiological Reviews, IX, 3, pp. 399-431.

Donnan, F. G. (1928). The Mystery of Life. Nature, 122, pp. 512-514. http://dx.doi. org/10.1038/122512a0

Durán, R. (2013). Autoorganización y estructuras disipativas: la imagen de naturaleza en llya Prigogine [Tesis doctoral inédita]. Pontificia Universidad Católica de Valparaíso: Valparaíso.

Editorial: Life and Death [Editorial]. Nature, octubre 1928, p. 501. plo clásico de Bénard, este movimiento convectivo iba asociado a la formación de formas hexagonales en la superficie del líquido. Con la fotografía de estas figuras geométricas se presenta generalmente el experimento, pero estas figuras no son producto de la propia actividad del sistema, sino debidas a la tensión superficial (Durán, 2013, p. 144).

13. Sin embargo, el propio Prigogine reconoce que su teoría dista de ser una respuesta definitiva al problema de la vida. "La noción de estructura disipativa no es suficiente seguramente ella sola para explicar la vida. Otros elementos fundamentales deben intervenir para explicar por ejemplo la duplicación» (Prigogine, 1969, pp. 57-58).

14. «Para Prigogine lo vivo [...] es disipativo» (Stengers, 2003, p. 225).

15. Los ingredientes o las condiciones de la autoorganización son: inestabilidad debido a no-linealidades, en particular

Espinoza, R. (2006). Realidad y tiempo en Zubiri. Granada: Comares.

Glansdorff, P. y Prigogine, I. (1971). Thermodynamic Theory of Structure, Stability and Fluctuations. New York: Wiley.

Gracia, D. (1986). Voluntad de verdad. Para leer a Zubiri. Barcelona: Labor.

Jacob, F. (1973). La lógica de lo viviente. Barcelona: Laia.

Keller, E. F. (2000). Lenguaje y vida. Metáforas de la biología en el siglo XX. Buenos Aires: Manantial.

Leclerc, I. (1956). Whitehead: La transformación del concepto de substancia. Convivium, 1, pp. 181-208.

Lwoff, A. (1962). Biological Order. Cambridge: MIT Press. http://dx.doi. org/10.5962/bhl.title.4514

Maturana, H. y Varela, F. (1994). De máquinas y seres vivos. Autopoiesis: la organización de lo vivo. Santiago de Chile: Editorial Universitaria.

Monod, J. (1985). El azar y la necesidad. Buenos Aires: Orbis.

Nicolis, G. y Prigogine, I. (1977). Self-Organization in Nonequilibrium Systems: From Dissipative Structures to Order through Fluctuations. New York: Wiley. auto-catálisis, irreversibilidad gracias al intercambio con el ambiente, fluctuaciones que gatillan el proceso, y uestructuras», resultado del proceso (Durán, 2013, p. 160).

16. Este es el esquema de reacciones del modelo irreal de Prigogine, el llamado «Brusselator»: (1) $A \rightarrow X$; (2) $B+X \rightarrow Y+C$; (3) $2 X+Y \rightarrow 3 X$; (4) $X \rightarrow D$. Para más detalles cf. Durán (2013, p. 159).

17. Las ecuaciones para el sistema anterior son: $\partial X / \partial t=k_{1} A-\left(k_{2} B+k_{4}\right) X+k_{3} X^{2} Y$; $\partial Y / \partial t=k_{2} B X-k_{3} X^{2} Y$.

18. Prigogine lo expresa refiriéndose a «la irreversibilidad como fuente de orden" (cf. Prigogine y Glansdorff, 1973; Durán, 2013, p. 276).

19. Respecto a la esencia y su relación con la noción de gen en biología cf. Vargas (2010).

20. Prigogine expresa la primacía de lo dinámico sobre lo estático en la morfogénesis (Prigogine, 2006, p. 85, p. 95).

Prigogine, I. (1945). Modération et transformation irréversible des systèmes ouverts. Bulletin de la Classe des Sciences. Académie Royale de Belgique, 31, pp. 600-606.

Prigogine, I. (1947). Étude thermodynamique des phénomènes irréversibles. Liège: Dunod, Paris et Desoer.

Prigogine, I. (1967). Introduction to Thermodynamics of Irreversible Processes. New York: Wiley.

Prigogine, I. (1969). Structure, Dissipation, and Life. En Marois, M. (ed.). Theoretical Physics and Biology. Amsterdam: North-Holland, pp. 23-52.

Prigogine, I. (1971). Dissipative Structure in Biological Systems. En Marois, M. (ed.). De la physique théorique à la biologie. Centre National de la Recherche Scientifique, pp. 162-183.

Prigogine, I. (1972). La termodinámica de la vida. En Prigogine, I. ¿Tan sólo una ilusión? Barcelona: Tusquets, pp. 299-325.

Prigogine, I. (1977). Physics and Metaphysics. Advances in Biological and Medical Physics, 16, pp. 241-265. http:// dx.doi.org/10.1016/B978-0-120052165.50021-9

Prigogine, I. (2004). ¿ ¿Tan sólo una ilusión?. Barcelona: Tusquets. 
Prigogine, I. (2006). El nacimiento del tiempo. Buenos Aires: Tusquets.

Prigogine, I. y Glansdorff, P. (1973). L'écart à l'équilibre interpreté comme une source d'ordre. Structure dissipatives. Bulletin de la Classe des Sciences. Académie Royale de Belgique, 59, pp. 673-702.

Prigogine, I. y Lefever, R., (1975). Stability and Self-Organization in Open Systems. En G. Nicolis, y Lefever, R. (eds.). Advances in Chemical Physics, XXIX. Wiley, pp. 1-28. http://dx.doi. org/10.1002/9780470143810.ch1

Prigogine, I. y Nicolis, G. (1971a). Biological Order, Structure and Instabilities. Quaterly Reviews of Biophysics, 4, pp. 107-148. http://dx.doi.org/10.1017/ S0033583500000615

Prigogine, I. y Nicolis, G. (1971b). Fluctuations and the Mechanism of Instabilities. En Marois, M. (ed.). From Theoretical Physics to Biology. Basel: Karger, pp. 89-100.
Prigogine, I. y Stengers, I. (1984). Order Out of Chaos. London: Flamingo.

Prigogine, I. y Stengers, I. (1994). La nueva alianza. Madrid: Alianza.

Schrödinger, E. (1944/1985). ¿Qué es la vida? Buenos Aires: Orbis.

Stengers, I. (1985). Les généalogies de l'auto-organisation. Cahiers du CREA, 8, pp. 7-104.

Stengers, I. (2003). Cosmopolitiques II. Paris: La Découverte.

Vargas, E. (2009). Estudio de la vida en Zubiri: un breve recorrido desde sus primeros cursos extrauniversitarios hasta Inteligencia sentiente. Pensamiento, 65 , 246, pp. 713-735.

Vargas, E. (2010). Relación entre el concepto de gen en la genética y el concepto de esencia en el libro Sobre la esencia de Xavier Zubiri. Cuadernos Salmantinos de Filosofía, 37, pp. 225-245.
Vargas, E. y Espinoza, R., (2013). Tiempo y edad biológica. Arbor, 189, 760, a22. http://dx.doi.org/10.3989/ arbor.2013.760n2008

Weiss, P. (1968). Dynamics of Development. New York: Academic Press.

Zubiri, X. (1980/2001). Inteligencia y razón. Madrid: Alianza.

Zubiri, X. (1996). Espacio. Tiempo. Materia. Madrid: Alianza.

Zubiri, X. (1998). Sobre la esencia. Madrid: Alianza.

Zubiri, X. (2001). Sobre la realidad. Madrid: Alianza.

Zubiri, X. (2006). Estructura dinámica de la realidad. Madrid: Alianza. 